prevalence of HIV testing and the disparities between HIV testing levels and self-reported HIV status in college students in Hong Kong.

Methods College students were recruited in four Hong Kong universities. Recruited subjects completed a structured questionnaire about their sexual behaviours. The prevalence of HIV testing and the inaccurate disclosure of HIV status (reporting an HIV-negative status without prior HIV testing) was estimated in sexually active students, and men who have sex with men (MSM) students, respectively.

Results Two hundred and fifty five students were included in the analysis. $16.1 \%$ of subjects were MSM. Overall, $81.6 \%$ of subjects reported that they had never had any HIV testing. $65.9 \%$ of subjects had inaccurate self-disclosure. Among subjects who had inconsistent condom use, only $20.2 \%$ of the subjects had HIV testing and $66.7 \%$ of the subjects had inaccurate self-disclosure. Among MSM students, only 36.6\% of the subjects had HIV testing before and $61.0 \%$ had inaccurate self-disclosure. Among MSM student who had inconsistent condom use, only $35.1 \%$ of the subjects had HIV testing and $62.2 \%$ had inaccurate self-disclosure.

Conclusion It was alarming to find the low prevalence of HIV testing but the high prevalence of inaccurate HIV status disclosure among sexually active college students, especially among MSM. The study findings raised the methodological issue of using a self-reported HIV status. Biological testing should be used whenever feasible, and public health interventions to promote HIV testing should be continued.

Disclosure No significant relationships.

\section{P128 HIV+ WOMEN 50+ STIGMATIZED AND DISCRIMINATED}

Chantal Mukandoli*. APAA (African In Partnership Against AIDS), Women Support, Toronto, Canada

10.1136/sextrans-2019-sti.304

Background Women living with HIV 50+years and older are facing, stigma while disclosing their status to their family friends, community, and partners. Women living with HIV are empowering and educating each other. Women face different issues (stress, depression, isolation, trauma or mental health issues). Disclosure of HIV status present challenges, there is need to support around women how to disclose and when. Women also are at higher risk of being criminalized by law for no-disclosure.

Methods We created social group in safe places for women living with HIV to come together to learn from each other regarding the issues they face. Women can speak about relationship, medications, health issues, and accessing services and program. Build each other's capacity skills. They gain a sense of connection through cooking, sewing, knitting. Peers who run the group are trained. The sessions were conducted in different sessions in the event. We administered pre- and postsession questionnaire to determine the effectiveness of the sessions. Demographic of those attending the group $87 \%$ identify as female, $13 \%$ identify as transwomen. Age $45-50+28 \%$, $50+72 \%$ attended group session.

Results (50) women attended this group sessions and received information and connected with other women living with HIV. 10 women reported that they have come out of isolation and are now support and mentoring other women in their communities. 5 women have been engaged in advocacy at the community and represent women in various working groups and committees.

Conclusion We are confident that social groups for women living with HIV help women to seek services, remain in care, reduce isolation and promote adherence. Meaningful involvement is more that inviting women at decision making tables but also addressing barriers that hinder women from engaging, addressing stigma, and other personal barriers that women might have.

Disclosure No significant relationships.

\section{P137 MODELLING FACTORS DETERMINING PAKISTAN'S HETEROGENEOUS HIV EPIDEMIC IN PEOPLE WHO INJECT DRUGS}

${ }^{1}$ Aaron Lim*, ${ }^{1}$ Adam Trickey, ${ }^{2}$ Laura Thompson, ${ }^{3}$ Tahira Reza, ${ }^{2}$ Faran Emmanuel, ${ }^{4}$ James Blanchard, ${ }^{1}$ Peter Vickerman. 'University of Bristol, Population Health Sciences, Bristol Medical School, Bristol, UK; ${ }^{2}$ University of Manitoba, Centre for Global Public Health, Department of Community Health Sciences, WINNIPEG, Canada; ${ }^{3}$ Centre for Global Public Health, Islamabad, Pakistan; ${ }^{4}$ University of Manitoba, Centre for Global Public Health Winnipeg, Canada

\subsection{6/sextrans-2019-sti.305}

Background Pakistan's HIV epidemics among people who inject drugs (PWID) vary widely across different cities and has increased dramatically over time. To help guide future HIV programming, we used statistical and mathematical modelling to identify possible causes for these differing HIV epidemics.

Methods Cross-sectional integrated biological and behavioural surveillance (IBBS) data describing high-risk behaviours and HIV status among PWID were collected by the Canada-Pakistan HIV/AIDS Surveillance Project (HASP) over five rounds from 2005-2017. We used regression analyses to identify citylevel associations between the prevalence of HIV infection and different risk behaviours. We then developed a dynamic HIV transmission model to reflect these associations. The model was calibrated to the IBBS dataset to estimate relative risks of HIV transmission due to each risk factor, and their respective population-attributable fractions (PAFs) over 10-years. Lastly, we investigated the prevention benefit that could be achieved if these behaviours were reduced across different settings to the lowest observed values.

Results Multivariable statistical analyses identified professional injecting use at last injection ('ProfInj') and heroin use within the past month ('HeroinUse') as key high-risk behaviours associated with city-level HIV infection, with HIV prevalence increasing by $4 \%$ and $2 \%$ for every $10 \%$ increase in the prevalence of each respective behaviour. Modelling projections estimated that ProfInj and HeroinUse increased the relative risk of HIV transmission by 7.9 (95\%CrI 4.6-14.0) and 2.2 (1.13.7) times, respectively, with 10 -year PAFs across all cities being 52\% (37-61\%) and 21\% (3-34\%), separately, and 61\% (51-66\%) combined. Lowering each behaviour's prevalence across all cities to the lowest observed prevalence (11\% ProfInj, 0\% HeroinUse) reduced overall relative HIV incidence by $43.9 \%(36.9-47.7 \%)$ and $44.1 \%(11.0-60.5 \%)$ over 10 -years, respectively.

Conclusion Pakistan's HIV epidemic is heterogeneous, with professional injecting and heroin use likely large contributors to the differing epidemics. Interventions focussed on these behaviours, especially professional injecting, could substantially reduce HIV incidence.

Disclosure No significant relationships. 